Artigo de Revisão

\title{
As políticas públicas e o enfrentamento da obesidade no Brasil: uma revisão reflexiva
}

\author{
Public policies and coping of obesity in Brazil: a reflective review
}

Daiani Cristina Rech ${ }^{1}$, Letícia Borfe ${ }^{1}$, Alessandra Emmanouilidis ${ }^{1}$, Edna Linhares Garcia ${ }^{1}$, Suzane Beatriz Frantz Krug ${ }^{1}$

${ }^{1}$ Universidade de Santa Cruz do Sul - UNISC, Santa Cruz do Sul, RS, Brasil.

Submissão:08/08/2016

Aceite: 02/09/2016

dainhar@hotmail.com

\section{RESUMO}

Justificativa e Objetivos: Atualmente, a obesidade é considerada um importante problema de saúde pública, pois sua prevalência configura-se em níveis alarmantes. Os custos atribuídos aos gastos com a obesidade são muito elevados e, a longo prazo, podem tornar-se empecilhos à sustentabilidade do tratamento dos indivíduos, tanto no âmbito público, quanto no privado. O objetivo foi refletir sobre a importância das Políticas Públicas voltadas ao enfrentamento da epidemia da obesidade e seus custos à saúde pública. Conteúdo: o tratamento das doenças relacionadas ao sobrepeso e a obesidade acarretam um número substancial nas despesas, sendo que no Brasil este dispêndio chega a ser de 5\% dos gastos destinados a saúde. Para enfrentar a epidemia da obesidade, as políticas públicas devem proporcionar ambientes estimuladores, que apoiem e mantenham padrões saudáveis de alimentação e atividade física, enfatizando que tais atitudes devem ir além de informar e educar a população. Diversas são as ações propostas pelo governo brasileiro para organizar políticas voltadas para a área de nutrição e atividade física que visam à prevenção e o tratamento da obesidade. No entanto, essas intervenções acabam atendendo situações imediatas e específicas, permanecendo o desafio para sejam consolidadas de forma universal. Conclusão: faz-se necessário um olhar mais ampliado na busca de políticas públicas realmente eficazes, pensadas em longo prazo e que possam envolver toda a população. Levar em consideração a conscientização sobre a prática de hábitos saudáveis, uma vez que intervenções pensadas na perspectiva da promoção da saúde terão impacto positivo nos recursos financeiros da saúde pública.

DESCRITORES: Políticas públicas. Obesidade. Promoção da saúde.

\section{ABSTRACT}

Background and objectives: Currently, obesity is considered a major public health problem because its prevalence set up at an alarming rate. The costs allocated to spending on obesity are very high and in the long run, can become obstacles to the sustainability of treatment of individuals, whether in the public, as in private. The objective was to reflect on the importance of public policies aimed at combating the epidemic of obesity and its costs to 
public health. Content: the treatment of diseases related to overweight and obesity entails a substantial number the costs, and in Brazil this expenditure is as high as 5\% of expenses related to health. To tackle the obesity epidemic, public policies should provide stimulating environments that support and maintain healthy patterns of diet and physical activity, emphasizing that such attitudes must go beyond informing and educating the population. There are several actions proposed by the Brazilian government to organize policies for the area of nutrition and physical activity aimed at prevention and treatment of obesity. However, these interventions end up taking immediate and specific situations, remaining the challenge to be consolidated universally. Conclusion: it is necessary to look at a broader search for truly effective public policies, designed in the long term and that may involve the entire population. Take into account the awareness of practicing healthy habits, since interventions designed to promote health perspective will have a positive impact on the financial resources of public health.

KEYWORDS: Public policies. Obesity. Health promotion.

\section{INTRODUÇÃO}

A obesidade é considerada um grave problema de saúde, sendo definida pela Organização Mundial da Saúde (OMS), como uma epidemia de escala global e um dos maiores desafios da saúde pública do início do século XXI. ${ }^{1}$ Pode ser caracterizada como doença crônica de múltiplas causas, em destaque as de caráter comportamental, como as alterações do padrão alimentar e sedentarismo. ${ }^{2}$

Estudos epidemiológicos atuais apontam para a crescente prevalência do sobrepeso e obesidade em idades cada vez menores e para possíveis danos que esse aumento pode gerar à saúde. ${ }^{3,4}$ A obesidade é fator de risco para doenças como: hipertensão arterial, arteriosclerose, carcinomas, diabetes tipo 2, síndrome de insuficiência respiratória do obeso, embolismo pulmonar, insuficiência cardíaca, infertilidade, propensão a quedas, entre muitos outros. Estima-se que no Brasil, doenças desencadeadas pela obesidade, matam por ano, em torno de 80 mil pessoas. ${ }^{5}$ Além disso, as doenças relacionadas com o excesso de peso, entre os anos de 2008 e 2010, resultaram em um custo direto de R \$ 2,1 bilhões para o sistema de saúde pública do Brasil. ${ }^{6}$

Para que as crianças e os adolescentes adquiram um estilo de vida adequado na fase adulta, é preciso que, desde cedo, a prevenção esteja focada na adoção de hábitos saudáveis, prevenindo futuros problemas de saúde. ${ }^{7}$ Neste contexto, as políticas públicas precisam necessariamente atender a demandas coletivas que trabalhem na prevenção e no tratamento da obesidade, visando o esclarecimento da população quanto ao consumo de alimentos e bebidas, e propondo ações que estimulem a prática de atividade física, prevenindo e tratando esta condição que já é fenômeno mundial. ${ }^{8}$ A partir deste contexto, o objetivo do estudo é 
destacar a importância das Políticas Públicas voltadas ao enfrentamento da epidemia da obesidade e seus custos a saúde pública.

\section{MÉTODOS}

Trata-se de uma revisão de literatura com busca nas bases de dados Medline, Lilacs e Pubmed através dos descritores em saúde (DECs) a partir das seguintes palavras: políticas públicas, obesidade, promoção da saúde, relacionadas na língua portuguesa e inglesa, priorizando artigos publicados nos últimos cinco anos.

\section{RESULTADOS E DISCUSSÃO}

\section{A obesidade e os custos à saúde}

A obesidade é considerada uma condição crônica de origem multifatorial, sendo resultado da interação entre fatores ambientais, comportamentais e genéticos, que influenciam nas respostas individuais à dieta e atividade física. ${ }^{9}$ No Brasil, a mudança do perfil nutricional que se desenha nos últimos 30 anos resulta num cenário epidemiológico preocupante que revela a importância de um modelo de atenção à saúde, que incorpore ações de promoção da saúde, prevenção e tratamento da obesidade e de doenças crônicas não transmissíveis. ${ }^{10,11}$

No Brasil em 2012, a prevalência de obesidade na região Norte já atingia 17,9\% da população, no Sudeste $17,7 \%$ dos indivíduos era considerado obeso, na região Nordeste esse índice atingia $16,7 \%$ da população, no Sul 16,9\% de homens e mulheres eram obesos e 15,6\% da população eram consideradas obesa na região Centro-Oeste. ${ }^{12}$ Dados da Pesquisa de Orçamento Familiar 2002/03 mostraram que cerca de 40\% dos adultos no Brasil estão com excesso de peso, sendo que destes, $8,9 \%$ do sexo masculino e $13,1 \%$ do feminino, em que a ocorrência mais elevada de excesso de peso é encontrada na população de baixa renda, destaca-se ainda a estabilidade e tendência de declínio nos segmentos de elevada renda, com exceção da região Nordeste, na qual a obesidade continua emergindo. O maior número de mulheres com excesso de peso é observado nas áreas rurais de todo o país, o que é diferente apenas no Nordeste, onde a maior concentração se dá nas áreas urbanas. Já nos homens, as maiores prevalências são observadas nas áreas urbanas das regiões Sudeste, Sul e CentroOeste e nos estratos de maior renda. ${ }^{13}$

No que diz respeito à incidência mundial, a temática da obesidade é descrita como um 
fator de risco, pois está relacionada a diversos distúrbios prejudiciais à saúde, como metabólicos, endócrinos, cardiovasculares, pulmonares, gastrointestinais, psiquiátricos, hematológicos, além do desenvolvimento de certas neoplasias malignas, sendo assim, é considerada hoje um grave problema de saúde pública que traz sérios agravos para a vida dos sujeitos, o que justifica a necessidade de atenção a este problema social. ${ }^{6,10,14}$ Atualmente, o consumo alimentar de diversos países têm sofrido intensas e rápidas modificações com progressiva substituição da escassez pelo consumo excessivo de alimentos, denominando a transição nutricional, que por sua vez, é considerada um fenômeno que atinge de forma desigual a nível nacional e mundial, com predominância em áreas de significativa urbanização e maior renda. Em consequência disso, mudanças no padrão alimentar são consideradas causas centrais do aumento nas taxas de obesidade e doenças crônicas. ${ }^{15}$

A escolha alimentar é complexa, e pode variar devido às características ambientais e individuais, assim como fatores econômicos, tais como renda e preço, que são fatores importantes na escolha alimentar, em que o mecanismo de preços exerce caráter proibitivo sobre escolhas alimentares de modo inversamente proporcional ao nível socioeconômico do indivíduo. Ressalta-se o papel da economia na investigação do processo de seleção de alimentos para composição da dieta, destacando contribuições às políticas públicas em saúde pela análise de fatores associados ao comportamento alimentar populacional, sendo assim, a compreensão dos mecanismos que permeiam relações de consumo de alimentos deve-se, majoritariamente, aos estudos econômicos sobre demanda observando-se as deficiências na construção de alguns modelos de demanda alimentar, especialmente sob a ótica nutricional e do planejamento de políticas públicas. ${ }^{15}$

Para os diversos sistemas de saúde, o tratamento das doenças relacionadas ao sobrepeso e à obesidade, acarreta em um aumento substancial nos gastos de saúde. ${ }^{16}$ Os custos de diversos países atribuídos à obesidade são elevadíssimos, girando em torno de 2 a $7 \%$ dos gastos destinados à área da saúde. Conforme estimativa financeira da Força Tarefa Latino-Americana de Obesidade, no Brasil, os gastos com internação de indivíduos com doenças associadas à obesidade, chega a ser de 5\%. ${ }^{17}$ Entre os anos de 2008 e 2011, o gasto médio do Sistema único de Saúde (SUS) com o tratamento da obesidade foi de $\mathrm{R} \$$ 25.404.454,87, sendo constatado um aumento de $\mathrm{R} \$ 16.260 .197,86$ neste período. ${ }^{18} \mathrm{O}$ crescente número de casos de obesidade configura, no longo prazo, uma ameaça à sustentabilidade do tratamento desses indivíduos tanto no âmbito público quanto no privado, ainda mais que a grande maioria da população brasileira depende exclusivamente do Sistema 
Único de Saúde (SUS) e sabe-se que o financiamento desse sistema é complexo e os recursos limitados. ${ }^{16}$

O conhecimento dos gastos, por parte da saúde pública, com o tratamento da obesidade, faz-se necessário para que políticas públicas direcionadas a este problema sejam implementadas. ${ }^{18}$ Para tanto o Brasil baseia suas alternativas em formulações de ações que visam à prevenção e o tratamento e em relação às políticas públicas da saúde verifica-se que as intervenções buscam atender situações imediatas e específicas, especialmente as que possam afetar a aspectos econômicos ou sociais. ${ }^{17}$

O impacto de uma intervenção de promoção à saúde em uma ampla perspectiva, certamente poderá refletir nos gastos do SUS em relação às enfermidades e mortes evitáveis, na melhoria da qualidade de vida da população e na compreensão de que manter a saúde é uma tarefa coletiva que exige um esforço do indivíduo, da comunidade e do governo em torno de metas a serem alcançadas. ${ }^{11}$

\section{A obesidade e as políticas públicas no contexto brasileiro}

As políticas públicas voltadas para a saúde têm sido de grande importância para a população do país, mesmo ciente das dificuldades de sua plena implementação. Seu principal papel é de orientar para a melhora da qualidade de vida da população, estimulando a promoção, proteção e recuperação da saúde dos indivíduos e do coletivo. Segundo a Constituição Federal promulgada em 1988, essas políticas orientam-se pelos princípios da universalidade, equidade no acesso às ações e serviços, descentralização da gestão, integralidade do atendimento e na participação da comunidade na organização de um sistema único de saúde a nível nacional. ${ }^{11}$

Para enfrentar a epidemia de obesidade, as políticas públicas e ações intersetoriais devem proporcionar ambientes estimuladores, que apoiem e mantenham padrões saudáveis de alimentação e atividade física, enfatizando que tais atitudes deveriam ir além de informar e educar a população. ${ }^{17}$ Propiciando também um ambiente que estimule, apoie e proteja padrões saudáveis de alimentação e atividade física, sendo por meio de medidas fiscais, que tornem mais acessíveis os alimentos saudáveis, de normas que limitem a publicidade de alimentos não saudáveis e de intervenções no planejamento urbano que facilite a prática cotidiana de atividade física, com uma ação consistente e estruturada na afirmação de responsabilidades do poder público em políticas intersetoriais articuladas, criando espaços de troca e construção constante de saberes, linguagens e práticas, buscando o desenvolvimento pessoal e social, 
habilitando as populações para opções por uma vida mais saudável, significa fazer com que, em cada um dos microssistemas, sejam organizadas ações de Promoção da Saúde. ${ }^{11}$

Diversos planos e ações governamentais já foram implantados no país e que influenciaram de algum modo na política atual, destacando-se no período do Estado Novo (1937-1945) os conceitos sobre o papel do Estado na alimentação. A partir de 1986, também vem sendo realizadas as Conferências Nacional de Alimentação e Nutrição e Conferências Nacional de Segurança Alimentar. ${ }^{6}$

Por meio da lei n. ${ }^{\circ} 8.080 / 1990$ é regulado o SUS atuar na formulação e no controle das políticas públicas de saúde. ${ }^{10}$ Nesse contexto, surge a Política Nacional de Alimentação e Nutrição (PNAN, portaria n. ${ }^{\circ}$ 710/1999), que atesta o compromisso do Ministério da Saúde em controlar os males relacionados à alimentação e nutrição no Brasil, através da formulação de requisitos básicos para a promoção e a proteção à saúde, ${ }^{11,17}$ possibilitando assim o processo de construção do empreendimento "Estratégia Global para Alimentação Saudável, Atividade Física e Saúde", que tem como principais objetivos: estabelecer um conjunto de orientações e recomendações para políticas e ações nas áreas da saúde, agricultura e desenvolvimento social; recomendar ações sobre as escolhas alimentares mais saudáveis dos indivíduos e ainda prever ações de caráter regulatório, fiscal ou legislativo, que modifiquem o ambiente de modo a tornar essas escolhas factíveis. ${ }^{6}$

Em 2003, com o intuito de garantir a todos os brasileiros acesso a uma alimentação adequada, à sobrevivência e à saúde em termos de quantidade, qualidade e regularidade foi implantado o Programa Fome Zero. ${ }^{6}$ Mais tarde, aprovada pela Portaria no 687/2006, a Política Nacional de Participação Social (PNPS), a qual transita entre o conjunto das ações e programas voltados à saúde, denota que com a sua implantação o protagonismo do processo de promoção da saúde, passa a ser dos profissionais e usuários do sistema. ${ }^{17}$

Devido os altos índices de sobrepeso e obesidade em crianças e adolescentes em idade escolar no Brasil e no mundo, o governo brasileiro lançou no ano de 2007, por meio do decreto presidencial no 6.286/2007, o Programa Saúde na Escola (PSE), uma ação interministerial entre os Ministérios da Saúde e Educação integrante dos planos específicos da PNPS, que tem como finalidade o desenvolvimento de ações de prevenção, promoção e atenção à saúde de crianças e adolescentes de cinco a dezenove anos no ambiente escolar, focando suas ações nos alunos de escolas da educação básica da rede pública de ensino. ${ }^{17}$

A escola é reconhecida como local privilegiado para promover a saúde, contribuindo com efeitos positivos na mudança de padrão de consumo e atividade física dos escolares, observando-se modificações dos hábitos de vida saudáveis. Outro espaço importante para a 
inserção das ações para saúde na Atenção Básica são os polos de Academias de Saúde. ${ }^{19} \mathrm{O}$ Programa Academia de Saúde, lançado em 2011 pelo Ministério da Saúde, objetiva contribuir para a promoção da saúde pública, incentivando a prática regular da atividade física e com a inserção de profissionais dos NASF (Núcleo de Apoio a Saúde da Família), que realizarão ações que atendam às demandas do seu território, propor também ações de promoção da alimentação saudável. ${ }^{20}$

A implantação de um sistema onde possa ser realizado o diagnóstico para adequado mapeamento da situação e das reais necessidades alimentares da população, dos mecanismos e das estruturas já disponíveis para ações de intervenção, em uma perspectiva de médio e longo prazo, depende do estabelecimento de políticas públicas concebidas em uma perspectiva de continuidade e com a importante e ativa participação da sociedade. ${ }^{21}$ Dada à complexidade da obesidade, é enriquecedor o trabalho interdisciplinar, onde é estudada e discutida a saúde da população. Este trabalho em equipe é importante para que se possa compreender de forma integral os sujeitos e os condicionantes da obesidade, ressaltando a crescente necessidade de políticas públicas eficazes para amenizar os processos de adoecimento e exclusão, causados pela obesidade. ${ }^{6}$

Portanto, faz-se necessário uma visão mais ampliada na busca de políticas que atentem para o controle da obesidade, propondo ações e estratégias de prevenção que abordem de forma conjunta a obesidade e outros problemas relacionados a peso e alimentação com foco em nutrição, atividade física, imagem corporal, influência da mídia e autoestima. ${ }^{8}$

\section{CONCLUSÕES}

Atualmente, o tema acerca de Políticas Públicas voltadas a prevenção da obesidade é amplamente discutido, onde muitas ações são pensadas por parte dos órgãos governamentais, a fim de propor meios de prevenção à doença e promoção à saúde através de programas educativos, campanhas e eventos que estimulem a participação da sociedade em geral.

Em decorrência da obesidade ser uma doença multifatorial, com múltiplas consequências e danos a saúde, tanto a curto como em longo prazo, deve-se pensar em ações amplas e integradas, com caráter intersetorial, envolvendo estratégias na área da saúde, educação, cultura, entre outras.

As Políticas Públicas que atentam a prevenção da obesidade devem estar centradas no bem-estar do indivíduo, envolvendo principalmente ações que estimulem a aquisição de hábitos saudáveis. Nesse sentido, se faz necessário idear meios de estimular a prática de 
atividades físicas, envolvendo todas as faixas etárias e todas as classes sociais. Também é de suma importância planejar intervenções que contemplem o prazer de uma alimentação saudável, garantindo a saúde plena de toda população.

\section{REFERÊNCIAS}

1. WHO. World Health Organization. Obesity: preventing and Managing the Global Epidemic. Report of a WHO Consultation. Geneva. WHO Technical Report Series n. 894, p. $1-12,2004$.

2. Beja A, Ferrinho P, Craveiro I. Evolução da prevenção e combate à obesidade de crianças e jovens em Portugal ao nível do planejamento estratégico. Rev Port Saúde Pública 2014; 32 (1): 10-17.

3. Daraki V, Georgiou V, Papavasiliou S, et al. Metabolic Profile in Early Pregnancy Is Associated with Offspring Adiposity at 4 Years of Age: The Rhea Pregnancy Cohort Crete, Greece. PLOS ONE 2015; 10 (5): 1-18. DOI: http://dx.doi.org/10.1371/journal.pone.0126327

4. Moselakgomo VK, Toriola AL, Shaw BS, et al. Índice de massa corpórea, sobrepeso e pressão arterial em escolares na província de Limpopo, África do Sul. Rev Paul Pediatr 2012; 30 (4): 562-569.

5. Santos AM, Sherer PT. Reflexões acerca das políticas públicas no enfrentamento a obesidade no Brasil. Sociedade em Debate 2011; 17 (1): 219-236.

6. Codogno JS, Turi BC, Sarti FM, et al. The burden of abdominal obesity with physical inactivity on health expenditure in Brazil. Motriz: Rev Educ Fis 2015; 21 (1): 68-74. DOI: http://dx.doi.org/10.1590/S1980-65742015000100009

7. Franco M. Prevention of childhood obesity in Spain: a focus on policies outside the health sector. Gaceta Sanitaria 2010; 24 (1): 49-55. DOI: http://dx.doi.org/ 10.1016/j.gaceta.2010.09.014

8. Freitas LKP, Cunha Junior AT, Medeiros HJ. Obesidade em adolescentes e as políticas públicas de nutrição. Cienc Saude Colet 2014; 19 (6): 1755-1762. DOI: http://dx.doi.org/10.1590/1413-81232014196.12382013 
9. Zhao J, Grant SFA. Genetics of Childhood Obesity. J Obesity 2011; (2011): 1-9. DOI: http://dx.doi.org/10.1155/2011/845148

10. Dornelles AD, Anton MC, Pizzinato A. O papel da sociedade e da família na assistência ao sobrepeso e a obesidade infantil: percepção de trabalhadores da saúde em diferentes níveis de atenção. Saúde e Sociedade 2014; 23 (4): 1275-1284. DOI: http://dx.doi.org/10.1590/S0104-12902014000400013

11. Reis CEG, Vasconcelos IAL, Barros JFN. Políticas públicas de nutrição para o controle da obesidade infantil. Rev Paul Ped 2011; 29 (4): 625-633.

12. Rede Interagencial de Informação para a Saúde. Indicadores básicos para a saúde no Brasil: conceitos e aplicações [Internet]. Brasília: Organização Pan-Americana da Saúde; 2008 [citado 2015 ago 25]. 349 p. Disponível em: http://tabnet.datasus.gov.br/tabdata/livroidb/2ed/indicadores.pdf.

13. Wanderley EM, Ferreira VA. Obesity: a plural perspective. Ciência \& Saúde Coletiva 2010; 15 (1): 185-194. DOI: http://dx.doi.org/10.1590/S1413-81232010000100024

14. Arslan N, Erdur B, Aydin A. Hormones and cytokines in childhood obesity. Indian Pediatrics 2010; 47: 829-839. DOI: http://dx.doi.org/10.1007/s13312-010-0142-y

15. Sarti MF, Claro RF, Bandoni DH. Contributions by food demand studies to the development of public policies in nutrition. Cad Saúde Pública 2011; 27 (4): 639-647.

16. Bahia LR, Araújo DV. Impacto econômico da obesidade do Brasil. Revista HUPE 2014; 13 (1): 13-17. DOI: http://dx.doi.org/10.12957/rhupe.2014.9793

17. Pimenta TAM, Rocha R, Marcondes NAV. Políticas Públicas de intervenção na obesidade infantil no Brasil: uma breve análise da Política Nacional de Alimentação e Nutrição e Política Nacional de Promoção da Saúde. UNOPAR Científica Ciências Biológicas e da Saúde 2015; 17 (2): 139-146.

18. Mazzocante RP, Moraes JFVN, Campbell CS. Gastos públicos diretos com a obesidade e doenças associadas no Brasil. Rev Ciências Méd 2012; 21 (1-6): 25-34.

19. Jaime PC, Silva ACF, Lima AMC, et al. Ações de alimentação e nutrição na atenção básica: a experiência de organização no Governo Brasileiro. Rev Nutri 2011; 24 (6): 809-824. DOI: http://dx.doi.org/10.1590/S1415-52732011000600002 
20. BRASIL. Portaria no 719, de 07 de abril de 2011. Diário Oficial [da] República Federativa do Brasil, Brasília, DF, n. 68, p.52, 08 abr. Seção 1, pt. 1.

21. Domeme SMA. Indicadores nutricionais e políticas públicas. Estudos avançados. 2003; 17 (48): 131-135. DOI: http://dx.doi.org/10.1590/S0103-40142003000200011 